\title{
Open code is not enough: towards a replicable future for geographic data science
}

Levi John Wolf* (University of Bristol, UK)

Sergio J. Rey (University of California, Riverside)

Taylor M. Oshan (University of Maryland, College Park)

(*corresponding: levi.john.wolf@bristol.ac.uk)

Submission to Conceptualizing a Geospatial Software Institute

Workshop 3: Strategic Plan and Governance of GSI

Open science practices are a large and healthy part of computational geography and the burgeoning field of spatial data science. In many forms, open geospatial cyberinfrastructure adheres to a varying and informal set of practices and codes that empower levels of collaboration that are impossible otherwise. Often these practices are formalized, such as the Open Source Initiative's definition of "open source" [1] or Richard Stallman's "Four Freedoms" [2]. In practice, however, open science generally \& open geographic data science in particular has been largely characterized by Stallman's latter three freedoms:

1. The freedom to study how the program works and change it.

2. The freedom to redistribute copies so you can help others.

3. The freedom to distribute copies of your modified versions to others.

Pathbreaking work in geographical sciences has explicitly brought these concepts into focus for our current model of open science in geography [3-6]. In practice, however, these blend together into a somewhat ill-advised but easy-to-use working definition of open science: you know open science when you see it (on GitHub).

However, open science lags far behind the needs revealed by this level of collaboration. Software freedoms mean very little in practice if they are not exercised. That is, offering scientific software under permissive licenses for free on the internet is (surprisingly) not a cure-all for open science. What good is the ability to modify someone else's R package if it's not intelligible? Scientific source code is rarely "self documenting," no matter how many literate programming frameworks or packages are used to embed expository discussion inside of programs. So-called "write-only software," designed only to finish a specific academic project, is unfortunately common. And, while academics are not (and, indeed, must never be) full research software engineers, some middle ground must be found.

Despite the freedom to edit $\&$ redistribute scientific software, thorough and effective documentation is simply not done at the cutting edge of scientific work. The cost of communicating complex code knowledge is often too high; the difficulty of writing good code documentation tends to be much more difficult than writing good code itself. Finally, the mathematics and statistical content of new scientific work is often itself difficult to understand. It may be the case that only a small set of authors understand the grounds of the work in the first place, suggesting that the ability to see, study, \& modify the source code has incredibly limited practical relevance.

Further (and possibly more concerning), current challenges to replication in science imply a different set of problems that require a different set of solutions than the nominal provenance of software freedoms. Shallow replicability, the ability to run the code used in an analysis on the same data and achieve the same result, only ensures Stallman's "Zeroth" freedom to run the program as you wish, for any purpose. More complicated concepts in deep replicability, involve the ability to transport ideas 
or conclusions from study to study; the "didactic relevance" [7] of a finding provides it with general relevance. This requires open science to be much more than push-button repeatability or open source; we must also actively ensure that software freedoms are accessable to other scientists. Thus, it is not enough to create open scientific software, we must also strengthen the open science community.

As a new Co-Maintainer of the Python Spatial Analysis Library, my colleagues and I have implemented practices for the cultivation \& maintenance of an open source community of practice, united around common standards and agreements about code quality, style, and documentation. In this talk, I will discuss a few of the best practices we, as a project, have developed, including:

1. Using a "developer contract" to provide consistency across aligned (but separate) projects.

2. Instituting active, robust scientific code review for papers, programs, and pedagogical material.

3. Creating easy, effective, and open technical spaces for contributors to write and maintain documentation for fellow developers.

4. Building space, tools, and practices to help junior developers get invested and involved.

5. Developing mentoring so interested users can eventually become developers themselves.

6. Ensuring each problem is solved exactly one time.

7. Providing simple, effective, and usable documentation for external scientific users.

The practices we have developed are, by no means, the only ones possible for open replicable science. However, we hope that by sharing them with Workshop 3: Strategic Plan \& Governance of GSI, we can instil the important message, that open science is not enough to ensure reproducible science; we also must have a sustainable \& open scientific community.

[1] Open Source Initiative, The Open Source Definition. https://opensource.org/osd. Accessed: 9 October, 2018

[2] Stallman, Richard. What is Free Software? https://www.gnu.org/philosophy/free-sw.en.html Accessed: 9 October, 2018

[3] Sergio J. Rey and Luc Anselin, "PySAL: A Python Library of Spatial Analytical Methods," The Review of Regional Studies 37, no. 1 (2007): 5-27;

[4] Rey, Sergio J. "Open regional science." The Annals of Regional Science 52, no. 3 (2014): 825-837.

[5] Rey, Sergio J. "Show Me the Code: Spatial Analysis and Open Source," Journal of Geographical Systems 11, no. 2 (June 2009): 191-207, https://doi.org/10.1007/s10109-009-0086-8;

[6] Rey, Sergio J. et al., "Open Geospatial Analytics with PySAL," International Journal of Geo-Information 4 (2015): 815-836.

[7] Ron Johnston, "On the Nature of Explanation in Human Geography," Transactions of the Institute of British Geographers 5, no. 4 (1980): 402-412. 